\title{
SCG0018-4854: a young and dynamic compact group *
}

\section{l. Kinematical analysis}

\author{
V. Presotto ${ }^{1,2}$, A. Iovino ${ }^{2}$, E. Pompei ${ }^{3}$, and S. Temporin ${ }^{4}$ \\ 1 Universitá degli Studi dell'Insubria, Via Valleggio 11, 22100 Como, Italy \\ e-mail: valentina.presotto@brera.inaf.it \\ 2 INAF - Osservatorio Astronomico di Brera Via Brera 28, 20122 Milan, Italy \\ 3 European Southern Observatory (ESO) Alonso de Cordova 3107, Santiago, Chile \\ ${ }^{4}$ Institut für Astro- und Teilchenphysik, Universität Innsbruck, Technikerstrasse 25, 6020 Innsbruck, Austria
}

Received Month Day, Year; accepted Month Day, Year

\begin{abstract}
Aims. It is widely recognized that processes taking place inside group environment could be among the main drivers of galaxy evolution. Compact groups of galaxies are in particular good laboratories for studying galaxy interactions and their effects on the evolution of galaxies due to their high density and low velocity dispersion. SCG0018-4854 is a remarkably high galaxy density and low velocity dispersion group with evidence of a recent interaction. It has been detected and analyzed at different wavelengths, but its kinematics has not yet been studied in detail.

Methods. We obtained VLT FORS2 optical observations and we present spectroscopic and photometric evidence of how dramatically galaxy interactions have affected each of the four member galaxies.

Results. We found peculiar kinematics for each galaxy and evidence of recent star formation. In particular, the gas and stellar radial velocity curves of two galaxies are irregular with a level of asymmetry similar to that of other interacting galaxies. We discovered the presence of a bar for NGC 92 therefore revising a previous morphological classification and we obtained spectroscopic confirmation of a galactic-scale outflow of NGC 89.

Conclusions. Peculiar kinematics and dynamic consideration lead to a rough estimate of the age of the latest interaction: $\tau \approx 0.2-$ 0.7 Gyr, suggesting that SCG0018-4854 is a young and dynamical group.
\end{abstract}

Key words. galaxies: clusters: individual: SCG 0018-4854 - galaxies: interactions - galaxies: kinematics and dynamics

\section{Introduction}

Compact groups (CGs) are among of the smallest and densest systems of galaxies of the universe, furthermore they are characterized by low velocity dispersions of the order of $\approx$ $200 \mathrm{~km} \mathrm{~s}^{-1}$ (Hickson et al. 1992). This is why they are excellent laboratories for studying galaxy interactions and their effects on the evolution of galaxies. Rubin et al. (1991) showed that $30 \%$ of spiral galaxies in CGs has distorted rotation curves and another $30 \%$ has irregular velocity patterns indicating that tidal interactions are frequent and persisting in CGs. Moreover, Mendes de Oliveira \& Hickson (1994) demonstrated that $43 \%$ of the galaxies in their compact group sample shows morphological and/or kinematical distortions indicative of interactions and/or mergers. Since these pioneering studies it has been clear that interactions play an important role in the evolution of groups. According to current dynamical models, galaxies belonging to groups should interact violently and merge on a relatively short time scale (Sulentic 1987; Barnes 1985; Governato et al. 1996). Yet, strong mergers in CGs are rare (Mendes de Oliveira \& Hickson 1994) and a lot of effort has been made to establish the origin and fate of such systems. Recent studies (Coziol \& Plauchu-Frayn 2007) have shown that galaxies in compact groups are more likely to merge under dry conditions, after they have lost most of their gas in interactions

* Based on observations collected at the European Southern Observatory, Cerro Paranal, Chile (proposal: 267.A-5722(A)) among galaxies. These hypothesis is corroborated by HI studies; Verdes-Montenegro et al. (2001) have found out perturbed distributions of neutral hydrogen in CGs. This results indicate that the evolution of CGs is mainly dominated by galaxy interactions, continuous tidal stripping and/or gas heating.

It is therefore interesting to reconstruct the evolution and the interaction history of a CG throughout the properties of its galaxy members and compare them with group properties as a whole. Kinematical and dynamical information are fundamental to separate between different interaction histories. Mendes de Oliveira et al. (1998) defined some kinematic indicators to distinguish between merging and interaction, such as misalignment between the kinematic and photometric axis of gas and stellar components, double gas components, warping and other peculiarities. However, there are still open questions about how many interactions take place and how strong and frequent they are.

In this work we focus on SCG0018-4854, a spiral-only compact group characterized by a really dense environment in which member galaxies have clearly interacted.

The plan for the article is the following. In Sect.2, we present the characteristics of SCG0018-4854. In Sect. 3, we describe our observations and the reduction process. In Sect. 4 we explain the methods used for our analysis. The results are presented in Sect. 5, followed, in Sect. 6, by a short discussion and our conclusions in Sect.7. 
V. Presotto et al.: SCG0018-4854: a young and dynamic compact group

\section{SCG0018-4854}

SCG0018-4854 is an extremely compact group of four latetype galaxies: NGC 92, NGC 89, NGC 87 and NGC 88. It was already listed in Rose's catalogue (Rose 1977) and it is part of the Southern Compact Group catalogue (Iovino 2000). It is located at $v \approx 3240 \mathrm{~km} \mathrm{~s}^{-1}$ which means $D \approx 45 \mathrm{~h}_{70} \mathrm{Mpc}$, adopting $h_{70}=\mathrm{H}_{0} / 70 \mathrm{~km} \mathrm{~s}^{-1} \mathrm{Mpc}^{-1}$. All galaxy members are within a projected distance of $\approx 1.6$ from the center of the group, where $1^{\prime \prime}$ corresponds to $225 \mathrm{pc}$. A possible fifth member, ESO198-G013, is located at a distance of 15' in the E direction. Furthermore, SCG0018-4854 has a remarkably low velocity dispersion, $\approx 120 \mathrm{~km} \mathrm{~s}^{-1}$ and high density, $4.88 \mathrm{gal} \mathrm{Mpc}^{-3}$ in log scale. Diffuse HI and X-ray emission has recently been detected confirming its bound nature (see Pompei et al. 2007; Trinchieri et al. 2008). The former data reveal a common HI envelope and a possible bridge between two galaxies, NGC 92 and NGC 88; according to Verdes-Montenegro et al. (2001) this HI distribution is typical of groups in phase $3 b$, i.e. with HI stripped from the galaxies and distributed in the common potential or in tidal tails. X-ray observation have revealed the presence of a hot intergalactic medium, $k T \approx 0.2 \mathrm{keV}$, in which the galaxies are embedded. The presence of a warm and hot intergalactic diffuse medium is important not only as an evidence of the gravitationally bound nature for SCG0018-4854, but also a proof of the galaxies interaction, even though it alone cannot explain tidal structures. Actually, all four close members show a disturbed morphology and a late-type appearance with intense activity, as testified by early spectroscopic data (Coziol et al. 2000). Here below we list the main properties of each galaxy while other information can be found in Table 1 .

- NGC 92 is a SAa pec starburst, also classified as a LINER by Coziol et al. (2000). It shows a ring of star-forming regions in its central part and it has an extended tidal tail in SE direction with many HII regions (Temporin et al. 2005). A peculiar extension in the tail direction is also detected in $\mathrm{X}$ ray (Trinchieri et al. 2008). Moreover, it has been suggested to contain a double nucleus (Danks \& Alcaino 1981). The galaxy has clearly undergone an interaction with one or several of the group members.

- NGC 89 is a SB0/a pec and it has a Sy2 nucleus and $\mathrm{H} \alpha$ emitting extra-planar features with a filamentary structure on both sides of the disk, including a $\sim 4 \mathrm{kpc}-$ long filament with a jet-like structure extending in NE direction (Temporin et al. 2005). It has also a ring of star forming regions around the nucleus. Interestingly, NGC 89 is the only galaxy of the group that was not detected in HI (Pompei et al. 2007). Probably it has lost its neutral gas after interacting with other group members and the IGM.

- NGC 88 is the third spiral, SBab pec, of the group with a LINER nucleus. It forms an HI bridge with NGC 92 (Pompei et al. 2007)

- NGC 87 is a nearly face-on irregular galaxy, based on its axis ratio. It has many distinct regions of strong star formation.

All these properties make SCG0018-4854 one of the few spiral-only and most active groups among all compact groups. It can be considered as the southern counterpart of HCG16, the most active group of all Hickson Compact Groups. This group is located at a similar redshift and it is composed of four peculiar late-type galaxies, as described by Hickson (1982), recently other three members have been added (Ribeiro et al. 1996). It has been detected both in HI and X-ray revealing the presence of a common medium (Verdes-Montenegro et al. 2001;
Belsole et al. 2003). All galaxy members show signs of interaction, such as tails and double nuclei, and different activities ranging from starburst to AGNs (de Carvalho \& Coziol 1999). The interaction affected also the velocity field of each galaxy, as shown by Mendes de Oliveira et al. (1998) who observed the presence of peculiar kinematics.

SCG0018-4854 has been detected and analyzed at different wavelengths, but its kinematics has not yet been studied in detail. In this paper we present first results of the kinematical analysis of this group and we compare its properties with those of HCG16. In a forthcoming paper (Temporin et al. in preparation) we will analyze in detail the photometric and morphological properties of each galaxy of SCG0018-4854, focusing on the likely age of the stellar populations and their correlation with morphological structures.

\section{Observation and data reduction}

The spectroscopic data have been obtained with the FORS2 spectrograph at the VLT-UT1, (Appenzeller et al. 1998), during an observing run in September 2001. The detector was a $2048 \times 2048$ px CCD with a scale of $0.201^{\prime \prime} \mathrm{px}^{-1}$ (we used the standard resolution collimator). We acquired spectra using the Spectroscopic Mask Mode (MXU). This kind of set-up offers the possibility to use a mask with slits of different length, width, shape and inclination suiting the observation demands. We used two different masks, one for all spectra along the major axis and the other for all spectra along the minor axis. We couldn't observe the major axis of NGC 88 due to overlaps of the slits in the same mask. We needed two different slits to cover the entire galaxy extent of NGC 92 (both axis) and NGC 89 (major axis). The data consist of four exposures of $2699 \mathrm{~s}$ for both masks, covering the major and the minor axis. All slits are 1" -wide and they have different length, position and inclination with respect to the dispersion axis. We used the GRISM-1400V with a dispersion of $20.8 \AA \mathrm{mm}^{-1}$, corresponding to $0.49 \AA \mathrm{px}^{-1}$. The grism peak is at $5200 \AA$ and the spectral coverage of each object spectra is $\approx 1000 \AA$ in the $4500-5900 \AA$ wavelength range according to the slit position with respect to the center of the CCD. Figure 1 and Tables 2, 3 show the slit positions and properties. A set of $\mathrm{He}-\mathrm{Cd}-\mathrm{Hg}-\mathrm{Ne}$ lamp spectra have been taken for each observing night. The average seeing was about $0.7^{\prime \prime}$ throughout our exposures. The individual frames were pre-reduced (bias subtraction, flat-field correction, etc.) using standard IRAF 1 image processing packages. The wavelength calibration was made using the IRAF TWODSPEC.LONGSLIT package. Our spectra have different inclination angles with respect to the dispersion axis. In order to rectify them we adapted the parameters of the task REIDENTIFY forcing it to identify and calibrate each lamp emission-line row by row. We verified our final wavelength solution using available skylines row by row. We adopt the rms of the $\lambda 5577 \AA$ skyline centroid distribution, $\approx 0.1 \AA$, as the calibration accuracy. The wavelength coverage for NGC 87 does not include any strong skyline so we verified the calibration directly on lamp spectra. We obtained the same accuracy as above. We found a systematic offset of $\approx 0.5 \AA$ in the calibration of all spectra along the major axis, probably due to instrument flexures (see Gallart et al. 2001). The final spectral resolution turned out

1 IRAF is distributed by the National Optical Astronomy Observatories, which are operated by the Association of Universities for Research in Astronomy, Inc., under cooperative agreement with the National Science Foundation. 
Table 1. SCG0018-4854 galaxy properties. RA and DEC data are taken from Nasa/IPAC Extragalactic Database, while morphology \& activity are taken from Pompei et al. (2007). References: (1) Danks \& Alcaino 1981; (2) Temporin et al. 2005 and (3) Pompei et al. 2007.

\begin{tabular}{lcccccc}
\hline \hline GALAXY & $\begin{array}{c}\text { RA } \\
(\mathrm{J} 2000.0)\end{array}$ & $\begin{array}{c}\text { DEC } \\
(\mathrm{J} 2000.0)\end{array}$ & $\begin{array}{c}\text { V } \\
\left(\mathrm{km} \mathrm{s}^{-1}\right)\end{array}$ & Morphology & Activity & Notes \\
\hline NGC 92 & $00: 21: 32$ & $-48: 37: 29$ & $3378 \pm 10$ & SAa pec & SBG & $\begin{array}{c}\text { tidal tail } \\
\text { double nucleus? }{ }^{1}\end{array}$ \\
NGC 89 & $00: 21: 24$ & $-48: 39: 55$ & $3287 \pm 10$ & SB0/a pec & Sy2 & H $\alpha{\text { galactic-scale extra-planar features }{ }^{2}}^{\text {no HI detection }^{3}}$ \\
& & & & & - \\
NGC 87 & $00: 21: 14$ & $-48: 37: 42$ & $3415 \pm 23$ & Irr & SFG & - \\
NGC 88 & $00: 21: 22$ & $-48: 38: 25$ & $3439 \pm 19$ & SBab pec & LINER & - \\
\hline
\end{tabular}

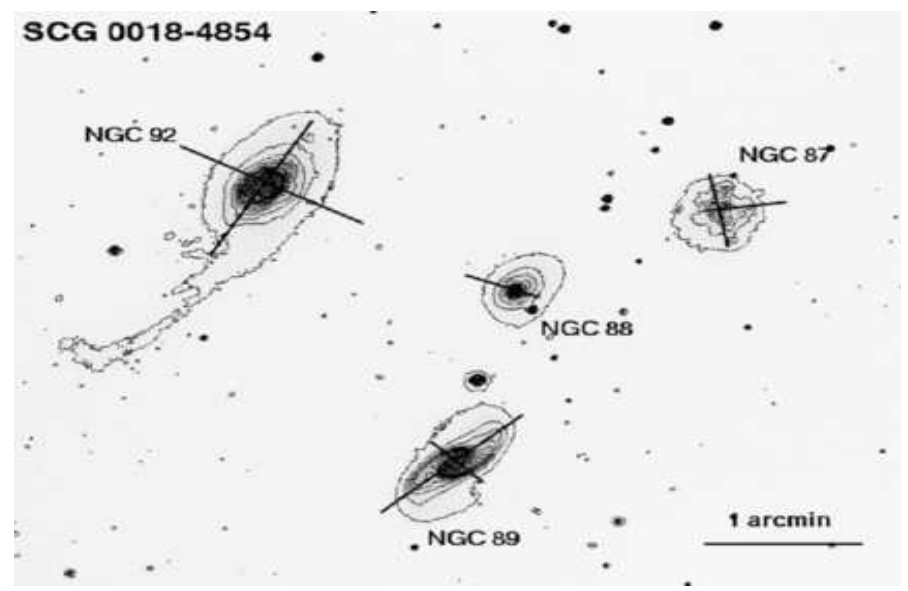

Fig. 1. $R$-band image of SCG0018-4854 with the slits and isointensity contours overlayed. North is up and east to the left.

Table 2. Slit properties of the mask along the major axis. $\mathrm{i}=$ inclination angle with respect to the dispersion axis, $\mathrm{PA}=$ position angle with respect to the $\mathrm{N}$ direction.

\begin{tabular}{cccccc}
\hline \hline Gal slit & $\begin{array}{c}\text { Width } \\
\left({ }^{\prime \prime}\right)\end{array}$ & $\begin{array}{c}\text { Length } \\
\left({ }^{\prime \prime}\right)\end{array}$ & $\begin{array}{c}\text { i } \\
(\mathrm{deg})\end{array}$ & $\begin{array}{c}\text { PA } \\
(\mathrm{deg})\end{array}$ & $\begin{array}{c}\lambda \text { coverage } \\
(\AA)\end{array}$ \\
\hline NGC 92 & 1 & $2 \times 39.8$ & 0 & -29 & $4845-5895$ \\
NGC 89 & 1 & $2 \times 35$ & -18 & -47 & $4530-5585$ \\
NGC 87 & 1 & 30.5 & 39 & 10 & $4450-5490$ \\
\hline
\end{tabular}

to be $2.25 \AA$ (FWHM of $\lambda 5577$ skyline), equivalent to a velocity of $\sigma \approx 51 \mathrm{~km} \mathrm{~s}^{-1}$. The sky subtraction was performed using a narrow region, $\approx 10 \mathrm{px}$, at both edges of the slit where there was minimum galaxy contamination. Unfortunately, we could not remove the sky from the spectrum along the major axis of NGC 87 because the galaxy covers the whole slit (a longer slit would have caused problems of overlap with the others). Our attempts to reproduce a good sky level failed, so we preferred not to subtract it. We extracted 1D spectra binning each 2D spectrum along the spatial direction in order to keep a signal-to-noise ratio $(\mathrm{S} / \mathrm{N}) \geqq 6$ at all radii. It should be noticed that in the central pixels we have a $S / N \geqq 100$, having such high $S / N$ data we just take into account the seeing when binning spectra in order to obtain uncorrelated spatial measure.

\section{Analysis method}

\subsection{Radial velocity curves}

Stellar kinematics was derived using the cross-correlation technique (Tonry \& Davis 1979) with the IRAF RVSAO package.
Table 3. Slit properties of the mask along the minor axis. $\mathrm{i}=$ inclination angle with respect to the dispersion axis, $\mathrm{PA}=$ position angle with respect to the $\mathrm{N}$ direction.

\begin{tabular}{cccccc}
\hline \hline Slit & $\begin{array}{c}\text { Width } \\
\left({ }^{\prime \prime}\right)\end{array}$ & $\begin{array}{c}\text { Length } \\
\left({ }^{\prime \prime}\right)\end{array}$ & $\begin{array}{c}\text { i } \\
(\mathrm{deg})\end{array}$ & $\begin{array}{c}\text { PA } \\
(\mathrm{deg})\end{array}$ & $\begin{array}{c}\lambda \text { coverage } \\
(\AA)\end{array}$ \\
\hline NGC 92 & 1 & $2 \times 39.9$ & 0 & 60 & $4620-5650$ \\
NGC 89 & 1 & 28 & -18 & 42 & $4850-5890$ \\
NGC 87 & 1 & 25 & 38.7 & -81 & $4450-5490$ \\
NGC 88 & 1 & 30 & 7.5 & 67 & $4620-5650$ \\
\hline
\end{tabular}

The cross-correlation was performed on a wavelength range of $\approx 900 \AA$ around the main absorption features of the $\mathrm{Mg}$ line triplet $(\lambda \lambda 5164,5173,5184 \AA)$. We decided to use the nuclear spectra of each galaxy as a template, thereby minimizing problems due to template mismatch. In this way we derived the relative velocities at various distances to the nucleus and so we constructed the stellar radial velocity curve. Error bars were derived by adding in quadrature the contributions from photon statistics, CCD read-out noise and calibration accuracy. It is worth noticing that stellar error bars are always larger than gas ones because the cross correlation was performed in a wavelength range with few absorption features. Furthermore those present in the spectra are not very intense, then the fit gives a large error.

In order to obtain the systemic velocity of each galaxy we performed a second cross-correlation with a radial velocity standard star extracted from the ESO archive. We used HD36003, a $\mathrm{K}$ star with an observing set-up that well matches that of our data. We assumed that the photometric center coincides with the dynamical one and we defined the systemic velocity of each galaxy to be that obtained with the cross-correlation with the standard star at the center of the galaxy along the major axis. All quoted velocities are corrected to the heliocentric reference system.

As already discussed, we could not measure the stellar kinematics of NGC 87 along the major axis because of the presence of the sky contamination. We decided to use the value of velocity at the center of the galaxy obtained from the minor axis as the systemic velocity.

The ionized gas kinematics was measured by a Gaussian fit to the main emission lines present in the spectra $(\mathrm{H} \beta$, [OIII] $\lambda \lambda 4959,5007)$ using the IRAF ONEDSPEC.SPLOT task. We derived the errors adding in quadrature both the calibration accuracy and the formal errors given as output by the fitting routine.

We discovered the presence of multiple components in the emission line profile of NGC 92 and NGC 89. In the case of NGC 92 we found two components and we used the deblending function of SPLOT to separate them, errorbars are the same as above. For NGC 89 the emission profile is quite complex, it 
could be the result of three or more overlapping components, see Sect. 5.2. We used the task NGAUSSFIT in order to better deblend them. In this case the error bars for gas velocities are obtained as the sum in quadrature of four different sources: (1) the finite slit width introduces an uncertainty of $1.2 \AA$. (2) the rms of the peak centroid among a sample of different fitting solutions obtained perturbing initial centroid guesses ranges between 0.01 and $0.5 \AA$ according to the component. (3) the error of the best fit ranges between 0.001 and $0.5 \AA$. (4) Finally the calibration accuracy is $0.1 \AA$. All these terms add up to a velocity error that ranges between 65 and $85 \mathrm{~km} \mathrm{~s}^{-1}$ according to the component.

\subsection{Asymmetry Parameter}

We were interested in revealing any sign of interactions and how much they affected the kinematical properties of the galaxies. As we said, most galaxies in groups have irregular or anomalous rotation curves so we tried to quantify any asymmetry. As an indicator we used the asymmetry parameter, $A P$, as described in (Dale et al. 2001):

$A P=\sum \frac{\|V(R)|-| V(-R)\|}{\sqrt{\sigma^{2}(R)+\sigma^{2}(-R)}} \times\left[\frac{1}{2} \sum \frac{\|V(R)|+| V(-R)\|}{\sqrt{\sigma^{2}(R)+\sigma^{2}(-R)}}\right]^{-1}$

This parameter takes into account how different is the behavior of the kinematically folded approaching and receding halves, so it quantifies possible asymmetries in the observed rotation curves. The error in $A P$ was estimated as follows: for each velocity measure, $V\left(R_{i}\right)$, we generated a normal velocity distribution centred at $V\left(R_{i}\right)$ and having a dispersion $\sigma$ equal to the errorbar $\sigma\left(\mathrm{R}_{i}\right)$ of $V\left(R_{i}\right)$; we drew a random value, $V_{\text {new }}\left(R_{i}\right)$, out of it. Then we estimated the new $A P$ using $V_{\text {new }}\left(R_{i}\right)$ and $\sigma\left(\mathbf{R}_{i}\right)$. We repeated this procedure in a $N=10000$ cycle and then we adopted as the $A P$ error the rms of the resulting $A P$ distribution, which resembles a normal one.

\section{Results}

\subsection{Kinematics of NGC 92}

In Fig. 2 we present the stellar (filled circles) and gas (open circles) radial velocity curves of NGC 92 along both the major (panel a) and the minor (panel b) axis. The kinematics along the major axis reveals two important features: i) at each radius the gas rotates faster than the stars. While the former reaches a maximal velocity $V_{\text {gas }} \approx 200-220 \mathrm{~km} \mathrm{~s}^{-1}$, the latter reach a maximum at $V_{*} \approx 140 \mathrm{~km} \mathrm{~s}^{-1}$. ii) While the stars show a regular radial velocity curve, the gas radial velocity curve is asymmetric with respect to the center of the galaxy. There is a steep rise of $V_{\text {gas }}$ up to $220 \mathrm{~km} \mathrm{~s}^{-1}$ within $1 \mathrm{kpc}$ in the NW direction, whereas on the other side the gas velocity reaches only $160 \mathrm{~km} \mathrm{~s}^{-1}$. We discover that the gas rotates also along the minor axis (see Fig. 2).

Moreover, the emission line profiles of $\mathrm{H} \beta$ and [OIII] $\lambda \lambda 4959,5007$ along the minor axis reveal the presence of a secondary component in addition to the main one. In Fig. 3 we show the position-velocity diagram with both the main (open circles) and the second (filled triangles) component of gas along the minor axis of NGC 92. Smaller panels show the zoom of 1D spectra in the range 5040-5080 $\AA$ of the [OIII] emission-line at each position where we were able to measure both components. Near the nucleus the second component was difficult to separate due to the broadening of the line and it appeared as an asymmetry of the profile. As we go far away from the nucleus a second peak

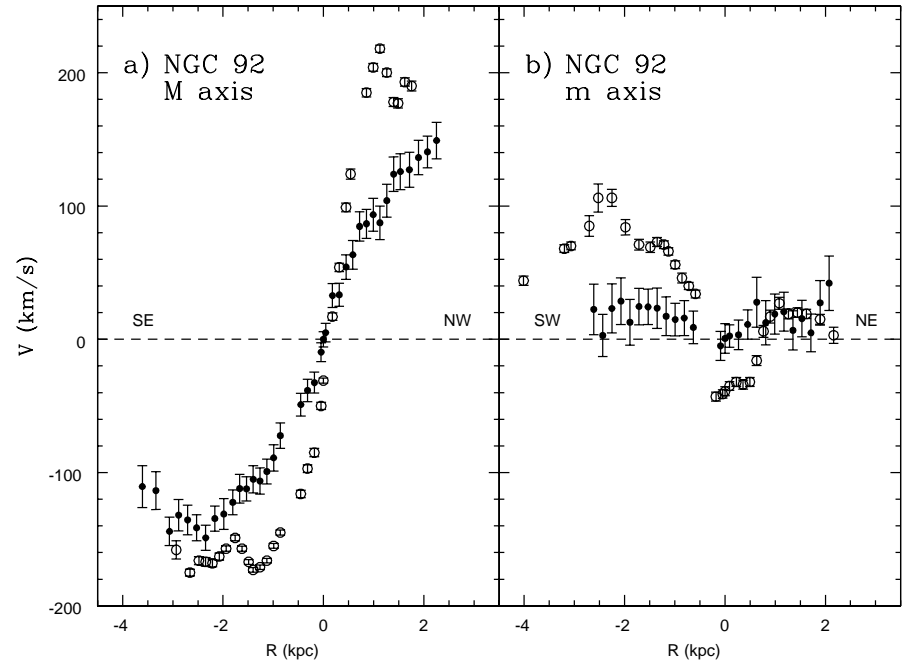

Fig. 2. Gas (open circles) and stellar (filled circles) kinematics along the Major (panel a) and the minor (panel b) axis of NGC 92.

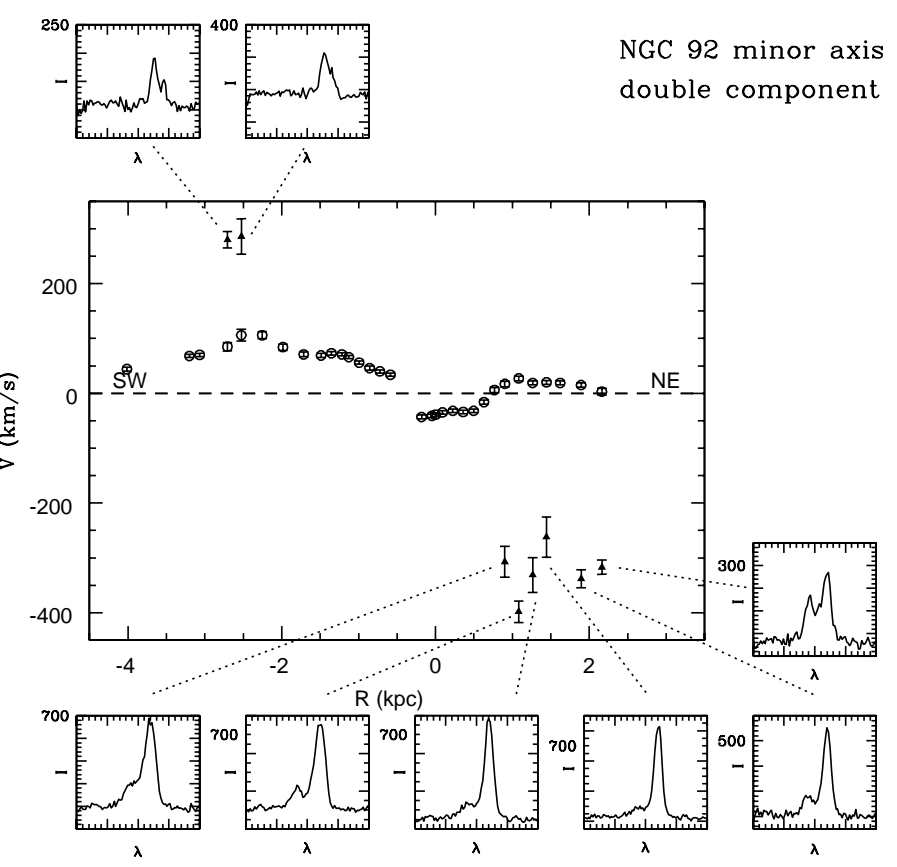

Fig. 3. Position-velocity diagram with both the main (open circles) and the second (filled triangles) component of gas along the minor axis of NGC 92. Smaller panels show the zoom of 1D spectra in the range 5040-5080 $\AA$ containing the [OIII] emissionline at each position where we were able to measure both components.

is clearly evident, as shown in the smaller panels of Fig. 3 At distances greater than $2.5 \mathrm{kpc}$ we do not detect the second component anymore.

The velocity gap between the components is $\Delta V \approx$ $200 \mathrm{~km} \mathrm{~s}^{-1}$ in the SW direction whereas it reaches $\Delta V \approx$ $350 \mathrm{kms}^{-1}$ in the NE direction. There can be two possible explanations for this double component: either we are looking at infalling gas with extra-planar motions (Fraternali \& Binney 2008) or the two components trace the well known eightshape due to the presence of a bar (Kuijken \& Merrifield 1995). To understand which of the two proposed explanations is 


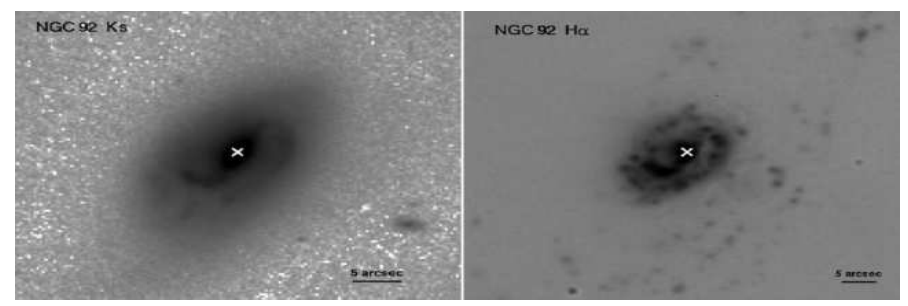

Fig. 4. Ks-band (left) and continuum-subtracted $\mathrm{H} \alpha+[\mathrm{NII}]$ (right) images of NGC 92. North is up and east to the left. The bar is well visible in the $K s$ image. Despite the different pixel scales of these images, it is clear that the $\mathrm{H} \alpha$ bright knot to the $\mathrm{SE}$ is the elbow of a spiral arm pointing towards the observer.

more likely, we inspected the $K s$-band and the continuumsubtracted $\mathrm{H} \alpha+[\mathrm{NII}]$ image 2 of NGC 92 (Hereafter $\mathrm{H} \alpha$ refers to $\mathrm{H} \alpha+[\mathrm{NII}])$. The $K s$-band image was obtained with SOFI at NTT and has a scale of $0.288^{\prime \prime} \mathrm{px}^{-1}$, whereas the $\mathrm{H} \alpha$ image was obtained with FORS2 and has a scale of $0.1^{\prime \prime} \mathrm{px}^{-1}$. These data will be presented and analyzed in detail in our forthcoming paper (Temporin et al. in preparation). Here we just report about a few morphological details of the inner regions of NGC 92 that are particularly useful for our interpretation of the kinematic features. The two images are shown in Fig. 4. The $K s$ image clearly shows the presence of a bar, thus corroborating the hypothesis that the second gas component along the minor axis is due to a bar. Also, from the comparison with the $\mathrm{H} \alpha$ image, it appears clearly that the $\mathrm{H} \alpha$ bright knot to the SE of the galaxy nucleus, thought to be a secondary nucleus, is actually the elbow of an inner proceeding spiral arm.

In Sect. 6]we will explore and discuss the implications of the presence of a bar for NGC 92.

In Fig. 5 we show the folding of the stellar (panel a) and the gas (panel b) radial velocity curves and their $A P$. The behavior of the stars looks quite regular, whereas the gas is much more perturbed. We obtain $A P$ values of $11 \% \pm 4 \%$ and $23 \% \pm 1 \%$ for the stars and the gas, respectively. In a recent work Pedrosa et al. (2008) used simulations to analyze the $A P$ of interacting and unperturbed galaxies in CGs and compare it with observations. They found $A P \approx 13-36 \%$ for the formers and $A P \approx 5-7 \%$ for the latter. These two ranges of values are in good agreement with our estimates for the observed velocity curves of the stars and the gas, respectively. Therefore, the stars of NGC 92 are unperturbed or only slightly perturbed while the gas $A P$ is in agreement with values obtained for other perturbed galaxies in groups. A mismatch between the two sides of the rotation curve was independently found also in a recent work by Torres-Flores et al. (2009) based on Fabry-Perot data. They also found signs of secondary kinematical components and a possible signature of non-circular motions. We refer to Sect. 6for a detailed discussion.

Additional emission lines are detected in the nucleus of NGC 92 and in the surrounding regions. These are HeI $\lambda \lambda$ 5015.68, 5875.6, [NI] $\lambda \lambda 5198.5,5200.7$ and [NII] $\lambda 5754.6$. Some of these lines require a sufficiently hard ionizing radiation (Osterbrock \& Ferland 2006) and, therefore, are normally found in regions photoionized by an AGN radiation, or in regions of strong and recent star formation, although most of them are generally difficult to detect because of their weakness. It is

2 The $[\mathrm{NII}] / \mathrm{H} \alpha$ emission line ratios evaluated in several regions of the galaxy by means of lower resolution spectra (not presented in this paper) together with the shape of the narrow-band filter transmission curve show that the [NII] emission lines contribute roughly $11.5 \%$ of the flux in the narrow-band image.

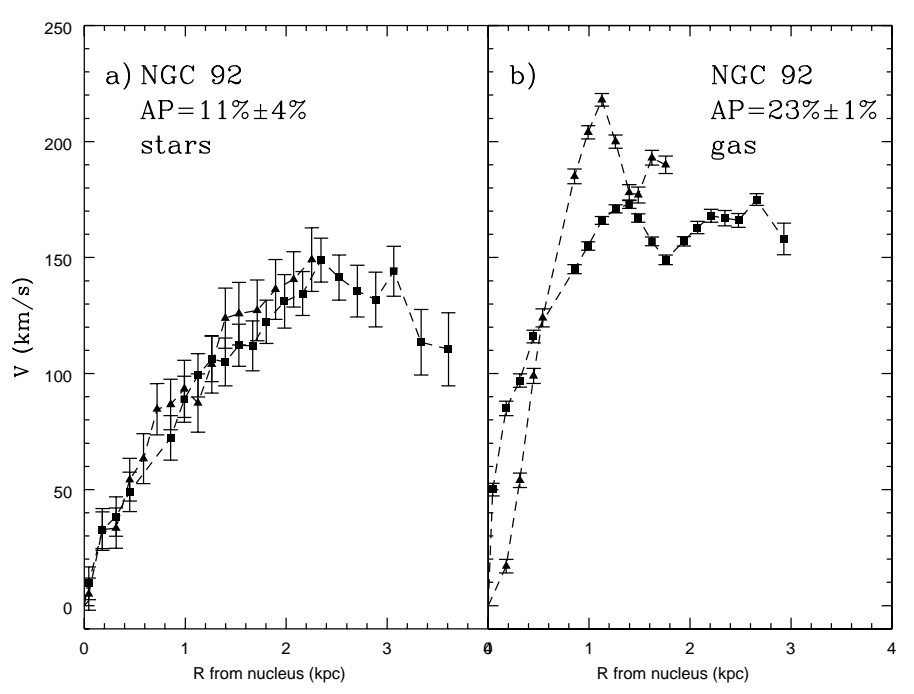

Fig. 5. Folding of the stellar (panel a) and the gas (panel b) radial velocity curve of NGC 92. Triangles are used for the receding side, in NW direction and squares for the proceeding side, in SE direction. The $A P$ values obtained for the stars and the gas are indicated.

interesting to note that the (usually very weak) HeI $\lambda 5015.68$ emission-line was detected only in correspondence of the elbow of the inner spiral arm, where additional data (not presented here) give evidence of the explosion of a supernova in year 1999 (Temporin et al., in preparation). The [NII] $\lambda 5754.6$ line - which is a well known electronic temperature indicator for the low ionization zones of gaseous nebulae, when combined with the more easily detectable [NII] $\lambda \lambda$ 6548,6583 emission-lines - is visible in the same spectrum and in the nuclear one. Finally, the [NI] doublet is detected in the nucleus and in all spectra out to 1.4 kpc NW and $2.1 \mathrm{kpc}$ SE of it along the major axis. Vaona et al. (in preparation), in a statistical study of the NLR of Sy2 galaxies extracted from the SDSS, find that the [NI] $\lambda \lambda 5198.5,5200.7$ doublet is detected in $8 \%$ of their sample ( 169 out of 2153 galaxies) and has a median ratio to $\mathrm{H} \beta$ of $\sim 0.14 \pm 0.06$. This value is consistent with what we observe in the nuclear region of NGC 92, which is classified as a LINER. Further out, the $[\mathrm{NI}] / \mathrm{H} \beta$ ratio assumes values of the order of a few $10^{-2}$, similar to those observed for HII regions in other LINER galaxies (e.g. Díaz et al. 2000). These [NI] lines are even observed in the presence of relatively weak radiation fields, like in the partially ionized zones of the Orion Nebula, and fluorescence is thought to play an important role in their excitation (Bautista 1999).

\subsection{Kinematics of NGC 89}

In Fig. 6 we present the stellar (filled circles) and gas (open circles) radial velocity curves of NGC 89 along both the major (panel a) and the minor (panel b) axis. Along the major axis we can measure velocities only out to $2 \mathrm{kpc}$ from the nucleus and both the gas and the stars reach a maximum $V \approx 100 \mathrm{~km} \mathrm{~s}^{-1}$. In the inner regions, $R<1 \mathrm{kpc}$, gas and stars show similar kinematics, while further out the gas has a slightly less regular pattern. In particular we notice that major discrepancies occur at $R \approx 1 \mathrm{kpc}$ in the NW direction. The gas kinematics is perturbed also along the minor axis: in the SW direction the gas velocity starts to increase at $\approx 1 \mathrm{kpc}$ and reaches $V \approx 90 \mathrm{~km} \mathrm{~s}^{-1}$ at $1.5 \mathrm{kpc}$. We suggest the possibility that the gas kinematics is affected by the 


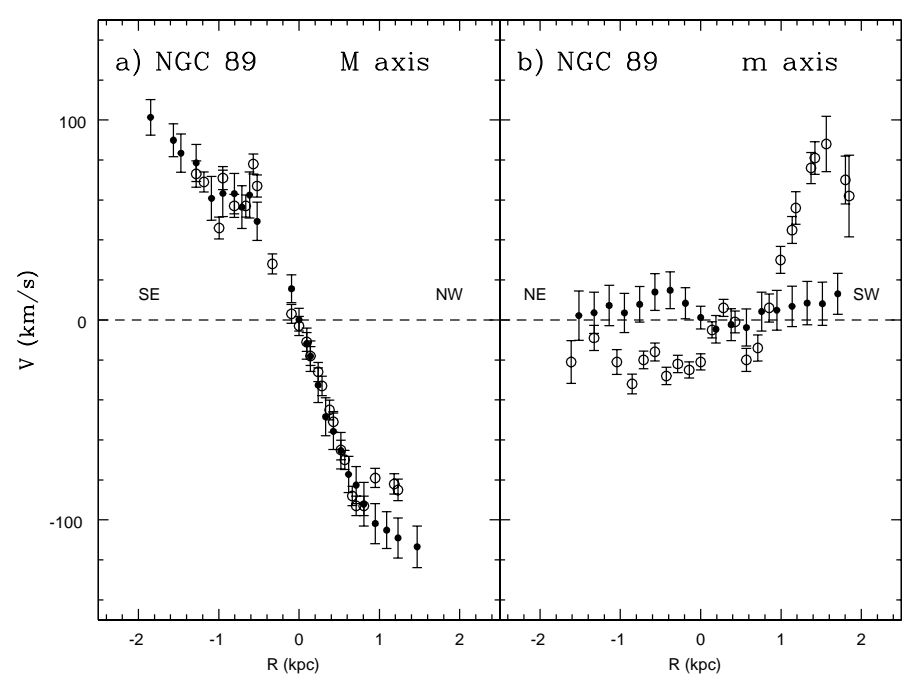

Fig. 6. Gas (open circles) and stellar (filled circles) kinematics along the Major (panel a) and the minor (panel b) axis of NGC 89.

central AGN and by the presence of the $\mathrm{H} \alpha$-emitting extraplanar gas (see Sect. 2).

We observe that the emission-line profile of [OIII] is asymmetric and irregular due to the presence of multiple components, at least three, in the nuclear region. This kind of profile is typically observed in the narrow line regions (NLR) of Sy2 galaxies. It is a common belief that photoionization and shocks play a dominant role in modifying the physical conditions of the gas (Dopita et al. 2002; Kraemer \& Crenshaw 2000; Veilleux et al. 2005), however the dynamics of the gas in the NLR is less certain. The presence of blue-shifts and red-shifts on either side of the nucleus indicates that rotation alone cannot explain the observed radial velocities. Emission-line profiles can be reproduced by different kinematic models: infall, outflow, rotation, etc. (Osterbrock \& Mathews 1986; Veilleux 1991). The general idea is that radial outflows play an important role.

The top panels of Fig. 7 show the position-velocity diagrams along the major (panel a) and the minor (panel b) axis for the main gaseous component (open circles), the [OIII] blueshifted component (filled triangles), and the [OIII] red-shifted one (filled squares). We observe non-circular motions of the gas with high velocities, $V \approx \pm 200 \mathrm{~km} \mathrm{~s}^{-1}$. In particular the redshifted component along the major axis has a regular pattern with high velocities within $150 \mathrm{pc}$ from the nucleus and then decelerates as we go farther away, $500 \mathrm{pc}$, in analogy to other cases of Sy2 NLR kinematics (see Das et al. 2006, and references therein). The multiple components can be seen more clearly in the spectral extractions around the nucleus. The bottom panels of Fig. 7 show the [OIII] emission-line profile obtained by summing 6 pixels $\left(1^{\prime \prime} .2\right)$ around the nucleus along the major (panel c) and the minor (panel d) axis as a function of velocity with respect to the systemic velocity of the galaxy. We fit the profile with a combination of three Gaussians: the main component (dotted line), the blue-shifted component (dot-dashed line) and the red-shifted one (dashed line). The blue-shifted component along the minor axis fails to fit two different bumps in the [OIII] emission-line profile and a fourth component is probably needed to better reproduce the [OIII] profile. We are able to perform the fit with four components in a satisfactory way only in the center. In Fig. 8 we show the comparison between the fit using three Gaussians (left) and the fit with four Gaussians (right). Residuals
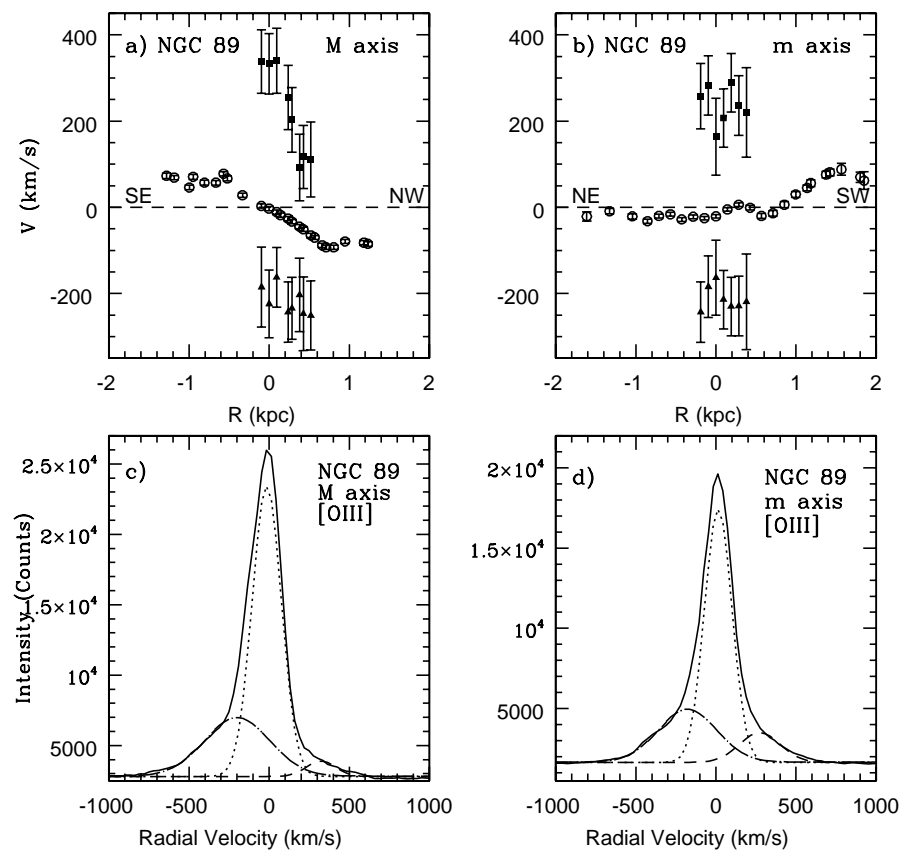

Fig. 7. NGC 89: position-velocity diagram along the major (panel a) and the minor (panel b) axis and the [OIII] emissionline profile obtained by summing 6 pixels $\left(1^{\prime \prime} .2\right)$ around the nucleus along the major (panel c) and the minor (panel d) axis as a function of velocity with respect to galaxy systemic velocity. We fit the profile with a combination of three Gaussians: the main component (open circles and dotted line), the blue-shifted component (filled triangles and dot-dashed line) and the red-shifted one (filled squares and dashed line).

of the fit are shown in the bottom panel in both cases. The choice of four Gaussians, the main component (dotted line), two blueshifted components (dot-dashed and short-long dashed line) and the red-shifted one (dashed line) gives better results, $\chi^{2} \approx 1.7$, with respect to that of three components, $\chi^{2} \approx 2.3$. The former residuals show a better agreement with the rms of the continuum as measured in two regions of 40 pixels at the edges of the emission-line profile. Although increasing the number of free parameters leads to better $\chi^{2}$, we should keep in mind that it also leads to a degeneracy of solutions. It is then difficult to establish which one is the most reliable, 3D spectroscopy with deeper integration time could help us to assess the possible presence of a fourth component.

However, it is interesting to note that this fourth component survives few pc away from the nucleus only in NE direction, that is the same direction of the galactic-scale $\mathrm{H} \alpha$ brightest filament. We cannot exclude that these two features are somehow linked to each other. The slit along the minor axis intercepts the jet-like filament in three different points. Therefore we are able to probe it spectroscopically and compare its kinematics with that of the adjacent gas.

In Fig. 9 we plot the measured kinematics of the additional gas components (filled triangles) together with the gas (open circles) and stars (filled circles) radial velocity curves. The kinematics of the extraplanar gas is clearly distinct from the disk gas kinematics. In fact, in the SW direction this gas component shows opposite velocity with respect to the disk gas. In the opposite direction we have two measures and there is a possible indication that the secondary gas component slows down as we go far away from the center of the galaxy. The decoupled kine- 


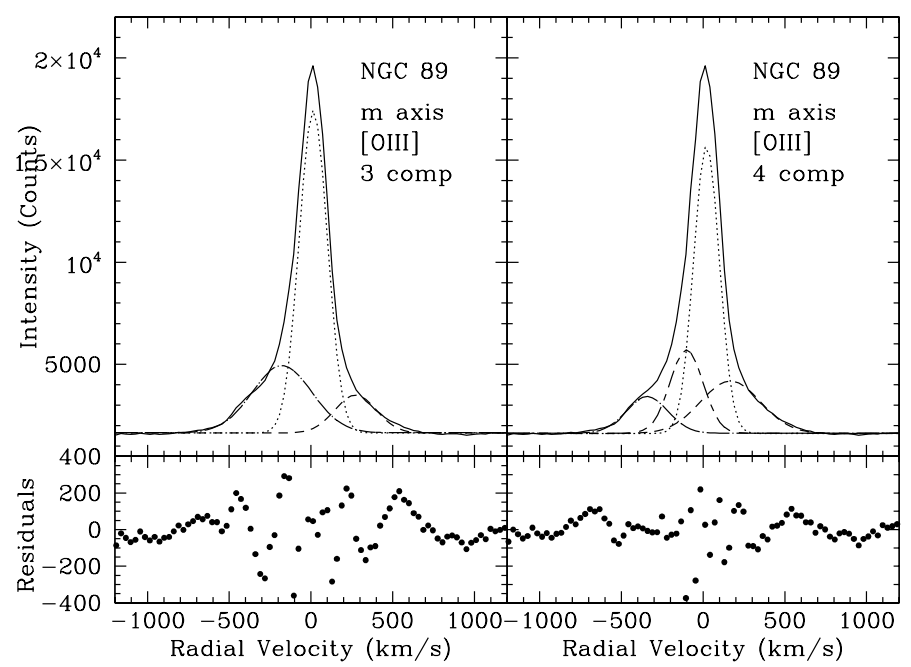

Fig. 8. Multiple component of the [OIII] emission-line profile along the minor axis of NGC 89. We compare the fit of the profile using a combination of three (left) or four (right) Gaussians. In the bottom panels we show residuals for both cases. The choice of four Gaussians, the main component (dotted line), two blue-shifted components (dot-dashed and short-long dashed line) and the red-shifted one (dashed line) gives better results.

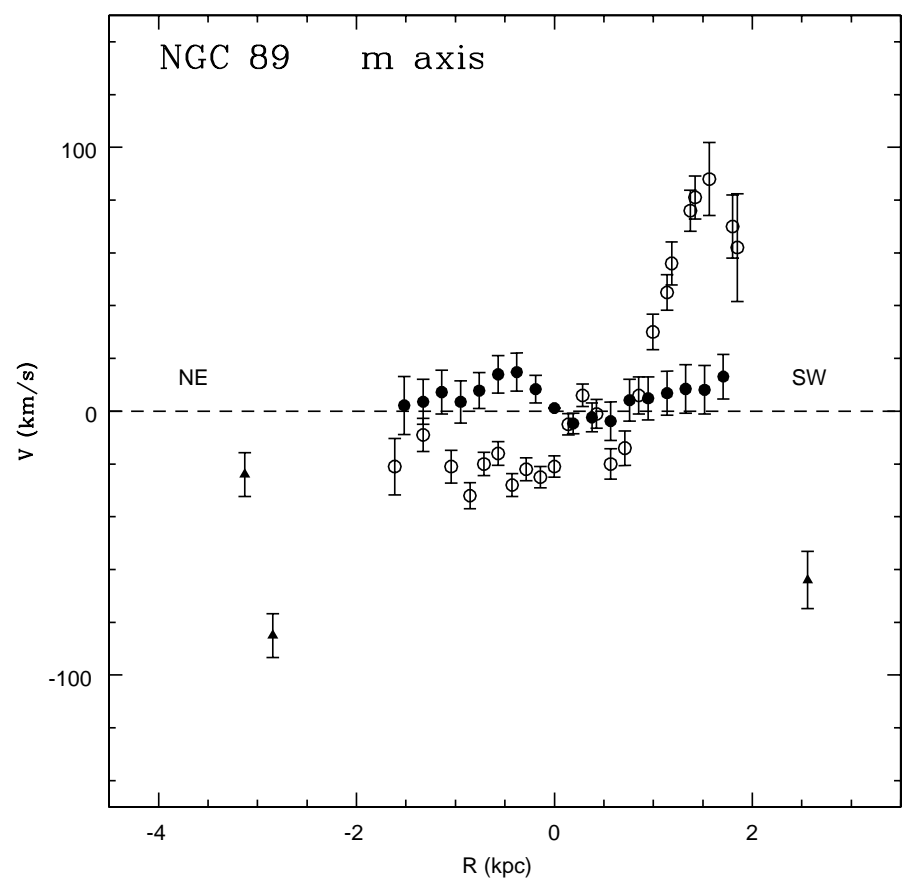

Fig. 9. Kinematics along the minor axis of NGC 89. Measured radial velocities for the primary gas component (open circles), the secondary gas component (filled triangles), and the stars (filled circles) are plotted. The secondary gas component is consistent with the presence of an outflow.

matics of the secondary gas component is compatible with the presence of an outflow. We discuss this possible interpretation in Sect. 6.3.

Once again we have estimated the asymmetry parameter. In Fig. 10 we show the folding of the stellar (panel a) and the gas (panel b) radial velocity curve and the relative $A P$. We obtain an $A P$ value of $33 \% \pm 6 \%$ and $24 \% \pm 4 \%$ for the stars and the gas respectively. These large values, as already mentioned in Sect.5.1

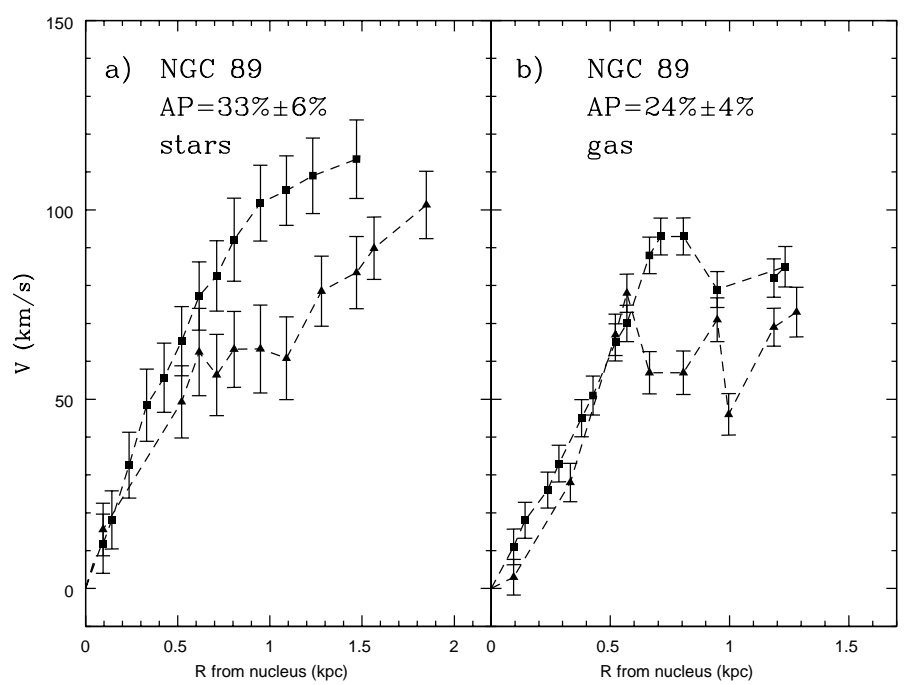

Fig. 10. Folding of the stellar (panel a) and gas (panel b) radial velocity curve of NGC 89 . Triangles are used for the receding side, in the SE direction and squares for the approaching side, in the NW direction. The values of the $A P$ for the gas and the stars are indicated.

are typical of strongly perturbed galaxies. We refer to Sect. [for a detailed discussion.

This galaxy as well shows many emission lines in the nuclear spectrum, in particular we reveal the presence of HeII 24685.75. This emission line can be observed in gas photoionized by the hard radiation field of an active galactic nucleus or it can be produced in the wind of Wolf-Rayet (W-R) stars (e.g. Crowther 2007), thus indicating a recent burst of massive star formation (Schaerer \& Vacca 1998). Usually, in W-R galaxies this emission-line is broad (although accompanied by an often difficult to disentangle narrow nebular component) and forms the so-called "W-R bump" in the spectrum together with other strong emission features (e.g. of CIII, CIV, and NIII) in the wavelength range $\lambda 4650-4690$ (Schaerer \& Vacca 1998, and references therein). Since the emission-line we observe is narrow, is not accompanied by a W-R bump and other typical features of W-R galaxies, and is visible only in the nuclear spectrum of NGC 89, we conclude that it is unlikely to be a Wolf-Rayet signature, but is rather the nebular emission produced by the ionizing radiation of the central AGN.

\subsection{Kinematics of NGC 87 and NGC 88}

In this Section we report the kinematical properties of the other two group members: NGC 87 and NGC 88 .

In Fig. 11 we present the stellar (filled circles) and the gas (open circles) radial velocity curves of NGC 87 along the minor axis (panel b), while along the major axis (panel a) we show only that of the gas (open circles). The lack of stellar kinematics along the major axis is due to problems in sky subtraction that made the use of XCSAO impossible, see Sect. 4 The gas shows a velocity gradient along both axes above which peculiar motions are superimposed. These peculiar motions belong to different regions of emission of the irregular galaxy. In both cases the velocity gradient is a regular trend with a significant velocity variation with respect to the errorbars and the velocity range of peculiar motions. Nevertheless, we cannot disentangle if the velocity gradient is associated to the whole galaxy or a few regions or if it is the evidence of a warp. The $\mathrm{HI}$ gas emission 


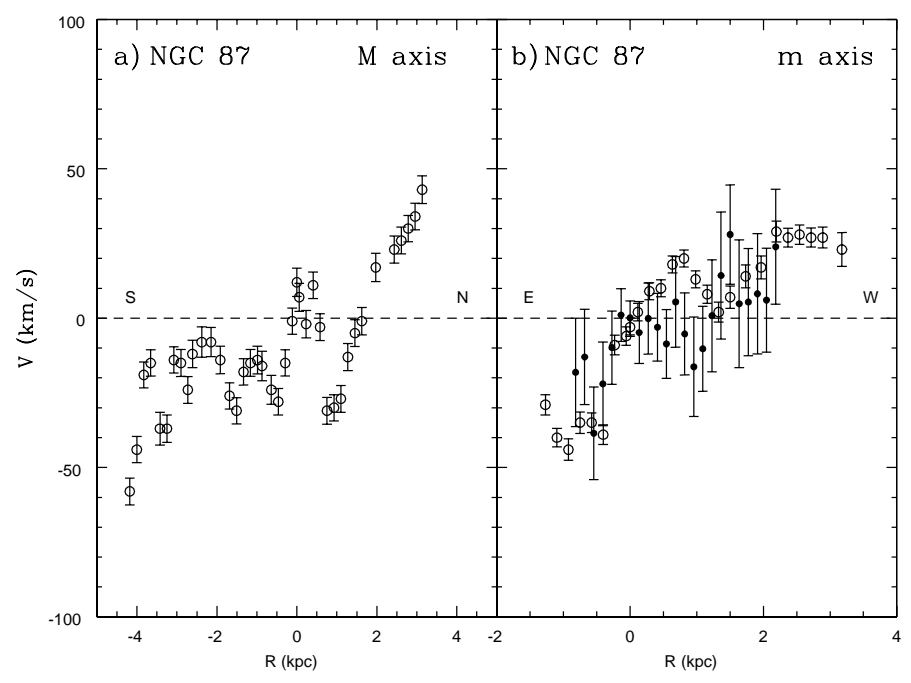

Fig. 11. Panel a - Gas (open circles) kinematics along the Major axis of NGC 87. Panel b-Gas (open circles) and stellar (filled circles) kinematics along the minor axis of NGC 87.

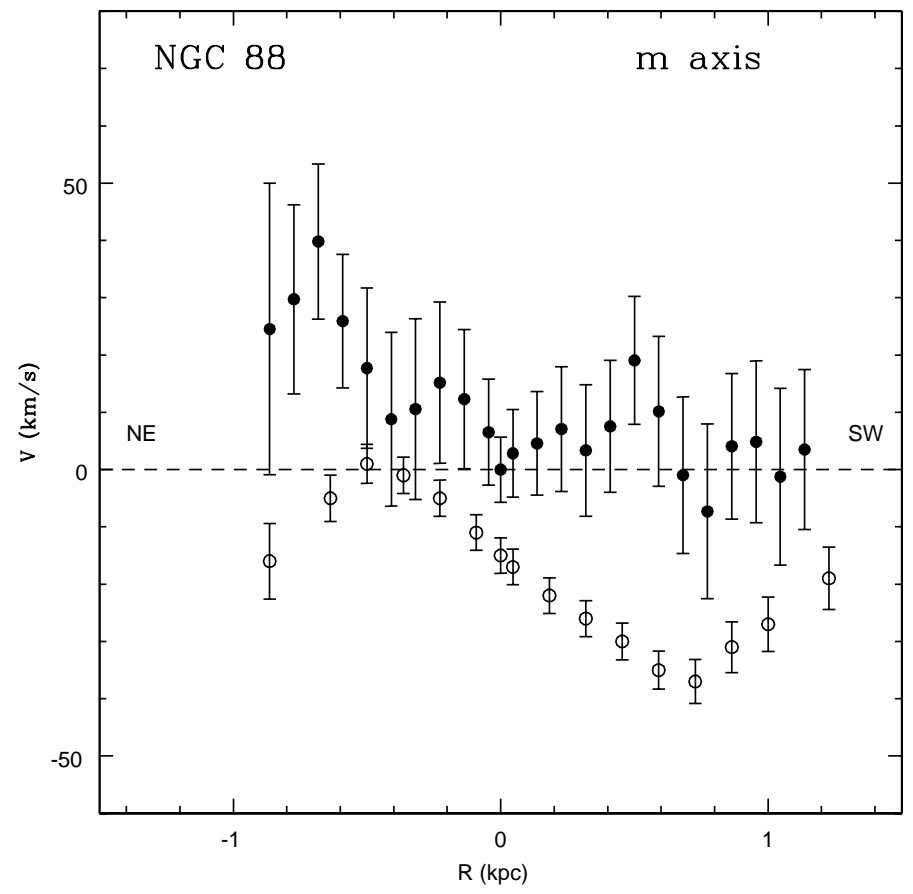

Fig. 12. Gas (open circles) and stellar (filled circles) kinematics along the minor axis of NGC 88.

is more extended than the optical light and the emission looks asymmetric; the HI velocity field does not show any significant gradient, as it would be for an almost face-on galaxy, except in the $\mathrm{N} / \mathrm{NE}$ corner at approximately the same position where the velocity gradient measured in the optical shows the largest variation.

In Fig. 12 we show the stars (filled circles) and gas (open circles) kinematics along the minor axis of NGC 88. The gas shows a sinusoidal pattern possibly indicating a regular rotation. Once again, the ionized gas is moving on extra-planar orbits. This kind of kinematics is widespread in galaxy bulges (Coccato et al. 2004) and it could be generated by a triaxial bulge or a bar. Kinematical information along the major axis is needed to find out which hypothesis is more reliable (Corsini et al. 2003).

\section{Discussion}

\subsection{Kinematical effects of the interaction on involved galaxies}

NGC 92 has clearly interacted with one or several group members as suggested by the extended tidal tail. We discuss now the kinematical effects of this interaction and which could be the other interacting galaxy/ies.

NGC 92 stellar radial velocity curve is only moderately perturbed, whereas the gas has an asymmetric radial velocity curve. The receding side has a steeper rise than the approaching one. Note that the approaching side is in the direction of the tidal tail, we suppose that the tail affects the gas kinematics keeping velocities at an approximately costant value of $160 \mathrm{~km} \mathrm{~s}^{-1}$. This is supported by HI data: neutral hydrogen velocities show a continuous trend between the tidal tail and the approaching side of the galaxy disk, clearly suggesting a physical link between gas, disk, and tail (Pompei et al. 2007). Arp 295 is a similar case of interacting galaxies with asymmetric rotation curves (Roche 2007). Also for Arp 295 the rotation curve rises slowly and monotonically on the side pointing towards the tail and the companion whereas on the other side it rises more steeply. Furthermore, a study of a sample of interacting galaxies reveals that there is a correlation between the SFR and perturbed kinematics (Keel 1993). The SFR increases as the rotation curves become more asymmetric from normal to disturbed rotation curves. Therefore, we expect to find enhanced star formation for NGC 92. Previous results from an $\mathrm{H} \alpha$ study of SCG0018-4854 reveal that the luminosity function of the HII regions of NGC 92 exhibits a considerable flattening at the high luminosity end (Temporin et al. 2005). NGC 89 and NGC 88 are candidate interacting galaxies with NGC 92, in fact we find irregular radial velocity curves and peculiar kinematics for both galaxies.

NGC 89 has perturbed stellar and gas radial velocity curves suggesting that it has interacted with other group members. Both gas and stars show lower velocities for the receding side with respect to the approaching one. This could be the result of different phenomena: (1) a disk warp, (2) streaming motions that are induced by interaction (3) composition of disk and spiral arm motions. 3D spectroscopy, which is currently unavailable for this galaxy, would help disentangling among these possibilities. Anyway, it is interesting to note that neutral and molecular hydrogen studies of this galaxy support the hypothesis that NGC 89 has interacted in the past with other group members. In fact NGC 89 has been completely stripped of its HI gas (Pompei et al. 2007) and CO observations show that it is perturbed also in its molecular gas distribution (Boselli et al. 1996). These facts suggest that also the perturbed kinematics of NGC 89 is a consequence of past interactions, the most likely perturber being NGC 92.

NGC 88 shows a sinusoidal pattern of the gas velocities probably due to a triaxial bulge or a nuclear bar. Both phenomena could be the result of an interaction. Moreover, there is an HI bridge between NGC 92 and NGC 88 (Pompei et al. 2007). Broad band observations show a wide stellar tail departing from this galaxy towards south-west (Temporin et al. in preparation).

The interaction process probably involves NGC 87 only partially: we found velocity gradients along both axis which could be the result of a slight interaction with other members. A stronger encounter would probably disrupt this galaxy due to its irregular morphology and lower mass. 


\subsection{The interaction strength and history of SCG0018-4854 galaxies}

The suggestion of a double nucleus for NGC 92 (Danks \& Alcaino 1981) could give rise to the hypothesis that this galaxy is a merger remnant. If this were the case, NGC 92 should have interacted violently with a companion galaxy in the past. Our results show that even if the interaction has been strong, we can exclude the hypothesis of a merger for NGC 92. The discovery of the nuclear bar rejected the hypothesis of a double nucleus. The comparison between $K s$-band and $\mathrm{H} \alpha$ images shows that the bright knot is actually the elbow of the inner proceeding spiral arm that departs from the nuclear bar. Therefore, NGC 92 should have interacted more recently and less strongly than the presence of a double nucleus could suggest.

However, it is well known that structures such as bars and rings could arise from galaxy interactions (Lindblad 1961; Noguchi 1987; Gerin et al. 1990). A direct tidal interaction acts as a destabilizing agent reducing the bar formation time-scale and accelerating the growth of the bar. Hence, we cannot exclude the possibility that the nuclear bar of NGC 92 be a product of its interaction with other group members and the presence of the tidal tail strengthens this hypothesis. 3D-spectroscopy could help to better understand the origin of the bar: e.g. an evidence of gas inflow could be a proof of a recent bar formation. Furthermore, a detailed study of the star formation along the bar could help to state whether the bar is linked to the interaction or not.

We try to quantify the interaction strength estimating the asymmetry parameter for NGC 92 and NGC 89 radial velocity curves. Cluster galaxies have a mean $A P$ of $12.6 \% \pm 1.2 \%$ (Dale et al. 2001) while, as we already said, galaxies in groups have larger values. We find $A P$ values in the range $11-33 \%$ which are in agreement with values of other perturbed and interacting galaxies in CGs. The gas is the first component that is affected in an interaction. Actually both NGC 92 and NGC 89 have been strongly perturbed in their gas kinematics. On the contrary, stellar kinematics are perturbed if the interaction has been violent. Therefore, since only NGC 89 shows a perturbed stellar kinematics, we speculate that the interaction was stronger for this galaxy than for NGC 92. Furthermore taking into account that NGC 89 is the only galaxy of the group depleted of HI we can not exclude a more complicated interaction history for this galaxy. NGC 89 could have interacted with the other groups members several times being NGC 92 the latest one. Otherwise NGC 89 could have joined the group only recently: the interaction with the group potential removed its gas and then it interacted with NGC 92. Hydrodynamical simulations could help us drawing the more likely scenario. Some caution in the interpretation is necessary, as recent studies show that the appearance of distortions in rotation curves is strongly dependent on the viewing angle (Kronberger et al. 2006). In any case there is a clear trend of increasing $A P$ for interacting galaxies. Pedrosa et al. (2008) analyzed the evolution of $A P$ in simulations of galaxy encounters with varying set-up. They showed that $A P$ rises at the peri-passage with values up to $\approx 20 \%$ and it starts decreasing only after $0.7 \mathrm{Gyr} h_{70}^{-1}$. In other words $A P$ could be used as a timer for recent interactions. Being SCG0018-4854 a compact group, its galaxies are supposed to encounter many times during their lives. We try to determine the age of the latest interaction among the galaxies of this group using the $A P$. Our estimated values of $A P$ for the gas and stars of NGC 92 and NGC 89 are large and suggestive of a recent interaction. Hence NGC 92 and NGC 89 should have interacted within the last 0.7 Gyr. This is only an upper limit as dynamical considerations on the tidal tail lead to a more recent interaction. A rough estimate of the age of the latest interaction is given by $\tau_{\text {tail }} \approx L_{\text {tail }} / V_{\text {flat }}$, where $L_{\text {tail }}$ is the tail extent, $\approx 30 \mathrm{kpc}$, and $V_{\text {flat }}$ is the velocity at which rotation curves become flat. In the tail direction $\mathrm{V}$ assumes an approximately constant value of $\approx 160 \mathrm{~km} \mathrm{~s}^{-1}$, which gives $\tau_{\text {tail }} \approx 190 \mathrm{Myr}$.

The above considerations lead to an estimated age of the latest interaction, $\approx 0.2 \mathrm{Gyr}<\tau_{\text {coll }}<0.7 \mathrm{Gyr}$, which is comparable with the crossing time of this group: 0.272 Gyr (Pompei et al. 2007). Further constraints on this matter will be obtained from the estimate of the age of the stellar populations through a detailed photometric analysis that is the subject of the already mentioned forthcoming paper.

\subsection{A galactic-scale outflow in NGC 89}

As mentioned in Sect. 2, the continuum-subtracted $\mathrm{H} \alpha$ image of NGC 89 shows the presence of ionised extra-planar gas (Temporin et al. 2005). This gas appears to be organised in a biconical structure departing from the central part of the disk and extending roughly perpendicularly to the disk plane on both sides of the disk. It has a filamentary structure and the prominent 4-kpc long jet-like structure is possibly just the brightest of these filaments. The origin and the nature of these $\mathrm{H} \alpha$ filaments cannot be fully established with the data presently available, but their geometry suggests the hypothesis of a galactic-scale outflow. Such an outflow could be driven by the combined action of the AGN and a central starburst, whose presence is indicated by a ring of bright $\mathrm{H} \alpha$ emitting regions in the central part of the galaxy. Indeed, the X-ray emission from NGC 89 was modelled as an absorbed AGN plus a diffuse emission coming from the central starburst (Trinchieri et al. 2008). However, we cannot exclude the possibility of an inflow. In order to discriminate between an inflow and an outflow beyond any doubt, we would need a detailed study of the interstellar absorption-line profiles, such as the NaI D $\lambda \lambda$ 5890, $5896 \AA$ (e.g. Rupke et al. 2005a).

Although another possibility would be the hypothesis that a minor merger has occurred in a recent past and a dwarf satellite has been cannibalised by NGC 89 , we find this interpretation less likely. In fact, at least with the existing data, we could not find any trace of a secondary nucleus in NGC 89. A search of the NED archive for the presence of dwarf satellites around the group revealed only the presence of two galaxies within an Abell radius with comparable redshift. This suggests that SCG00184854 is genuinely isolated. Still, we cannot exclude that NGC 89 might have had a dwarf satellite that was disrupted in a mergeer event. However, in the case of a minor merger we would expect to observe structures such has tidal streams forming loops and shells around the main galaxy or features resembling X-shaped jets, as it is the case for other systems that have been explained as the result of the disruption of a dwarf galaxy cannibalized by its more massive companion (e.g. Mrk 315 and NGC 1097 , see Ciroi et al. 2005; Higdon \& Wallin 2003). Instead, the $\mathrm{H} \alpha$ images show a wide distribution of extra-planar gas on both sides of the galaxy disk.

On the other hand, both AGN-driven and starburst-driven winds on galaxy scale have been observed in other Seyfert 2 and starburst galaxies both in the local Universe and at higher redshifts (see Veilleux et al. 2005, for a review). Powerful largescale outflows might be caused by strong starbust activity in the central regions of a galaxy with a consequent shock-heating and acceleration of ambient interstellar gas (e.g. Heckman et al. 
1990). Recent simulations of starburst-driven galactic winds reveal that optically emitting filaments can be formed by fragmentation and acceleration of a cloud into a supersonic wind (Cooper et al. 2008). When radiative cooling is taken into account, these fragments survive enough to generate strands and filaments downstream of the original cloud position like those seen in starburst-driven winds (Cooper et al. 2009). A well known case is that of M82, which shows optical filaments whose velocity increases with radius (Shopbell \& Bland-Hawthorn 1998). The observed morphologies and kinematics of outflowing gas on large scales reminds of the structures we observed in NGC 89. By analysing a sample of infrared luminous starbursts, Rupke et al. (2005b) found that superwinds are ubiquitous in these galaxies and in most cases the outflowing gas has velocities of $100-200 \mathrm{~km} \mathrm{~s}^{-1}$.

Bomans et al. (1997); Tschöke et al. (2001); Strickland et al. (2004) have shown the presence of a correlation between $\mathrm{H} \alpha$ outflow structures and X-ray features. Cooper et al. (2009) demonstrate also that the soft X-ray emission is due to mass ablated from the clouds and mixed into the surrounding gas. After $0.75 \mathrm{Myr}$ the fraction of gas mixed into the surrounding gas is $\approx 25 \%$. Hence, we would expect to observe some X-ray features correlated to the galactic-scale filaments of NGC 89. Trinchieri et al. (2008) did not detect any X-ray feature linked to the $\mathrm{H} \alpha$ emission. However, the total number counts of their $\mathrm{X}$-ray observations and the presence of two dominant components in the emission related to NGC 89, namely an absorbed AGN and an unresolved component from binary systems, would make it extremely difficult to detect a further faint component related to the low surface brightness $\mathrm{H} \alpha$ filaments that trace the extra-planar gas $\left(\right.$ Flux $\left._{H \alpha}=10^{-16}-10^{-17} \mathrm{erg} \mathrm{s}^{-1} \mathrm{~cm}^{-2}\right)$. Also, any interaction of the outflow with the IGM is expected to be very weak, since the IGM in which the members of SCG00184854 are embedded is cooler $(k T \approx 0.2 \mathrm{keV})$ and less dense $\left(n_{e} \approx 5 \times 10^{-3} \mathrm{~cm}^{-3}\right)$ than that typically found in other environments such as groups with a central elliptical galaxy or the cores of clusters (Trinchieri et al. 2008).

Our study of NGC 89 reveals a complex gas kinematics in the inner regions: emission line profiles have multiple components along both the major and the minor axis. We show evidences of at least two secondary components along both axis: one blue-shifted and the other red-shifted. This kind of composite motions are well known in literature for the NLR of Seyfert galaxies (Das et al. 2006, and references therein). However, a detailed kinematical model for the NLR is beyond the scope of this paper. The presence of a possible fourth component in the emission-line profile along the minor axis is intriguing as this component is visible only in the direction of the galacticscale extra-planar features. If indeed a galactic-scale outflow is present, we cannot exclude a link between such an outflow and the central engine.

In Sect 5.2 we probed the kinematics of the extra-planar gas in a few positions. The velocity difference of $\approx 60 \mathrm{~km} \mathrm{~s}^{-1}$ between the two measured positions in NE direction could be interpreted as a signature of the presence of two gas shells with different velocities due to the impact of the gas with the ISM. Moreover, when going far away from the nucleus along the minor axis we found that the gas is accelerated in the SW direction, consistently with the outflow interpretation.

Therefore, the combined information stemming from the kinematics and the geometry of the ionised extraplanar gas favours the outflow scenario. Marquez et al. (1998) studied a similar case: NGC 2992 is a Seyfert 1.9 galaxy with an emission arc, visible in [OIII] and $\mathrm{H} \alpha$, in interaction with its companion
NGC 2993. A radial outflow was added to the circular motion to account for the observed kinematics. They found largest differences with respect to the model when going at larger distances from the nucleus and suggested that these are kinematically distinct regions where the gas could be perturbed by the interaction of NGC 2992 with its close companion NGC 2993. This could be the case also for NGC 89 since the HI velocity field reveals that the tidal tail of NGC 92 is pointing towards the observer with a velocity comparable to that of NGC 89 (Pompei et al. 2007)

With the currently available data we are not able to establish the origin of the galactic-scale outflow. Integral-field spectroscopy would be useful to better understand the outflow kinematics and constrain its origin.

\subsection{Comparing SCG0018-4854 with HCG16}

The extreme physical properties of SCG0018-4854 make it a very interesting and rare group. It could be considered the southern counterpart of HCG16, the group with the highest concentration of late-type active and interacting galaxies in the nearby universe. Therefore, we compare its kinematic properties with those found in literature for HCG 16. The latest and more detailed kinematical study of HCG16 has been carried out by Mendes de Oliveira et al. (1998) using Fabry-Perot data. They found kinematic peculiarities for three galaxies out of the four originally listed in Hickson's catalogue (Hickson 1982). They found a non-axisymmetric rotation curve with a velocity discrepancy of $100 \mathrm{~km} \mathrm{~s}^{-1}$ between the two sides and a slow rise in the central region. They discovered the presence of a second component in the emission line profile of one galaxy. They detected severe warping of the kinematic major axis of two galaxies and finally the presence of a double nucleus and a peculiar bar-like structure. Based on these results, they suggested that major merger events have taken place in at least two out of four galaxies. Our study reveals that most of these kinematic peculiarities are also present in SCG0018-4854, confirming its nature of a relatively dynamically young and active $\mathrm{CG}$.

\section{Conclusions}

We have analyzed high signal-to-noise spectra along both the major and the minor axis of each galaxy of SCG0018-4854. Each galaxy shows disturbed or peculiar kinematics. Kinematic information is important in order to have some insight about the possible interaction history of the galaxies. Different interaction scenarios, depending on the strength and the geometry of the encounter and the morphological types of the interacting systems, will leave different signatures on the galaxy kinematics. Following Mendes de Oliveira et al. (1998) we found out signs of disturbed velocity fields, asymmetric rotation curves, multiple kinematic gas components, tidal tails and nuclear activity. All these characteristics suggest that at least three out of four galaxies of SCG0018-4854 have been involved in a strong and recent interaction. We derived some constraints for the age of the latest close interaction: $\approx 190 \mathrm{Myr}<\tau_{\text {coll }}<0.7 \mathrm{Gyr}$. The interaction has been strong enough to perturb the gas kinematics up to a level of $24 \%$ for the gas of the two main galaxies. These results are in agreement with the recent claim by Torres-Flores et al. (2009) that this group is in an advanced stage of interaction, based on the presence of young star forming regions and a candidate tidal dwarf galaxy identified in the UV in addition to the observed tidal tails. Finally, the results of our spectroscpic analysis are consistent with the hypothesis of a large-scale outflow in NGC 89. 
In contrast to its evolved properties as a group such as the high spatial density, the spatial distribution of $\mathrm{HI}$ and the presence of a common hot IGM, SCG0018-4854 is entirely composed of spiral galaxies and has a remarkably high fraction of active galaxies. Moreover, this study reveals kinematical signs of recent interactions among its members making SCG0018-4854 a dynamically young group although its global properties suggest a more advanced stage of evolution.

Given the estimated age of the latest interaction, we could say that we catch these galaxies in the act of interacting. What about the future of this group? Hydrodynamical simulations could help us to understand the fate of SCG 0018-4854, a really isolated interacting compact group.

Acknowledgements. We are grateful to the anonymous referee for his/her prompt and constructive comments that helped us to improve this paper. This research has made use of the NASA/IPAC Extragalactic Database (NED) which is operated by the Jet Propulsion Laboratory, California Institute of Technology, under contract with the National Aeronautics and Space Administration. V.P. is grateful for support from the short term studentship DGDF 09/39 awarded by the European Southern Observatory (ESO).

\section{References}

Appenzeller, I., Fricke, K., Fürtig, W., et al. 1998, The Messenger, 94, 1

Barnes, J. 1985, MNRAS, 215, 517

Bautista, M. A. 1999, ApJ, 527, 474

Belsole, E., Sauvageot, J.-L., Ponman, T. J., \& Bourdin, H. 2003, A\&A, 398, 1

Bomans, D. J., Chu, Y.-H., \& Hopp, U. 1997, AJ, 113, 1678

Boselli, A., Mendes de Oliveira, C., Balkowski, C., Cayatte, V., \& Casoli, F. 1996, A\&A, 314, 738

Ciroi, S., Afanasiev, V. L., Moiseev, A. V., et al. 2005, MNRAS, 360, 253

Coccato, L., Corsini, E. M., Pizzella, A., et al. 2004, A\&A, 416, 507

Cooper, J. L., Bicknell, G. V., Sutherland, R. S., \& Bland-Hawthorn, J. 2008, ApJ, 674, 157

Cooper, J. L., Bicknell, G. V., Sutherland, R. S., \& Bland-Hawthorn, J. 2009, ArXiv e-prints

Corsini, E. M., Pizzella, A., Coccato, L., \& Bertola, F. 2003, A\&A, 408, 873

Coziol, R., Iovino, A., \& de Carvalho, R. R. 2000, AJ, 120, 47

Coziol, R. \& Plauchu-Frayn, I. 2007, AJ, 133, 2630

Crowther, P. A. 2007, ARA\&A, 45, 177

Dale, D. A., Giovanelli, R., Haynes, M. P., Hardy, E., \& Campusano, L. E. 2001, AJ, 121, 1886

Danks, A. C. \& Alcaino, G. 1981, A\&A, 98, 223

Das, V., Crenshaw, D. M., Kraemer, S. B., \& Deo, R. P. 2006, AJ, 132, 620

de Carvalho, R. R. \& Coziol, R. 1999, AJ, 117, 1657

Díaz, A. I., Castellanos, M., Terlevich, E., \& Luisa García-Vargas, M. 2000, MNRAS, 318, 462

Dopita, M. A., Groves, B. A., Sutherland, R. S., Binette, L., \& Cecil, G. 2002, ApJ, 572, 753

Fraternali, F. \& Binney, J. J. 2008, MNRAS, 386, 935

Gallart, C., Martínez-Delgado, D., Gómez-Flechoso, M. A., \& Mateo, M. 2001, AJ, 121, 2572

Gerin, M., Combes, F., \& Athanassoula, E. 1990, A\&A, 230, 37

Governato, F., Tozzi, P., \& Cavaliere, A. 1996, ApJ, 458, 18

Heckman, T. M., Armus, L., \& Miley, G. K. 1990, ApJS, 74, 833

Hickson, P. 1982, ApJ, 255, 382

Hickson, P., Mendes de Oliveira, C., Huchra, J. P., \& Palumbo, G. G. 1992, ApJ, 399, 353

Higdon, J. L. \& Wallin, J. F. 2003, ApJ, 585, 281

Iovino, A. 2000, in Astronomical Society of the Pacific Conference Series, Vol. 209, IAU Colloq. 174: Small Galaxy Groups, ed. M. J. Valtonen \& C. Flynn, $25-+$

Keel, W. C. 1993, AJ, 106, 1771

Kraemer, S. B. \& Crenshaw, D. M. 2000, ApJ, 544, 763

Kronberger, T., Kapferer, W., Schindler, S., et al. 2006, A\&A, 458, 69

Kuijken, K. \& Merrifield, M. R. 1995, ApJ, 443, L13

Lindblad, P. O. 1961, Soviet Astronomy, 5, 376

Marquez, I., Boisson, C., Durret, F., \& Petitjean, P. 1998, A\&A, 333, 459

Mendes de Oliveira, C. \& Hickson, P. 1994, ApJ, 427, 684

Mendes de Oliveira, C., Plana, H., Amram, P., Bolte, M., \& Boulesteix, J. 1998, ApJ, 507, 691

Noguchi, M. 1987, MNRAS, 228, 635

Osterbrock, D. E. \& Ferland, G. J. 2006, Astrophysics of gaseous nebulae and active galactic nuclei, ed. G. J. Osterbrock, D. E. \& Ferland
Osterbrock, D. E. \& Mathews, W. G. 1986, ARA\&A, 24, 171

Pedrosa, S., Tissera, P. B., Fuentes-Carrera, I., \& Mendes de Oliveira, C. 2008, A\&A, 484, 299

Pompei, E., Dahlem, M., \& Iovino, A. 2007, A\&A, 473, 399

Ribeiro, A. L. B., de Carvalho, R. R., Coziol, R., Capelato, H. V., \& Zepf, S. E. 1996, ApJ, 463, L5+

Roche, N. D. 2007, Revista Mexicana de Astronomia y Astrofisica, 43, 179

Rose, J. A. 1977, ApJ, 211, 311

Rubin, V. C., Hunter, D. A., \& Ford, W. K. J. 1991, ApJS, 76, 153

Rupke, D. S., Veilleux, S., \& Sanders, D. B. 2005a, ApJ, 632, 751

Rupke, D. S., Veilleux, S., \& Sanders, D. B. 2005b, ApJS, 160, 115

Schaerer, D. \& Vacca, W. D. 1998, ApJ, 497, 618

Shopbell, P. L. \& Bland-Hawthorn, J. 1998, ApJ, 493, 129

Strickland, D. K., Heckman, T. M., Colbert, E. J. M., Hoopes, C. G., \& Weaver, K. A. 2004, ApJS, 151, 193

Sulentic, J. W. 1987, ApJ, 322, 605

Temporin, S., Ciroi, S., Iovino, A., et al. 2005, in Astrophysics and Space Science Library, Vol. 329, Starbursts: From 30 Doradus to Lyman Break Galaxies, ed. R. de Grijs \& R. M. González Delgado, 78P-+

Tonry, J. \& Davis, M. 1979, AJ, 84, 1511

Torres-Flores, S., Mendes de Oliveira, C., de Mello, D. F., et al. 2009, ArXiv e-prints

Trinchieri, G., Iovino, A., Pompei, E., et al. 2008, A\&A, 484, 195

Tschöke, D., Bomans, D. J., Hensler, G., \& Junkes, N. 2001, A\&A, 380, 40

Veilleux, S. 1991, ApJS, 75, 383

Veilleux, S., Cecil, G., \& Bland-Hawthorn, J. 2005, ARA\&A, 43, 769

Verdes-Montenegro, L., Yun, M. S., Williams, B. A., et al. 2001, A\&A, 377, 812 